\title{
Modeling the Temperature Distribution During Laser Hardening Process
}

\author{
Amir Mosavi1 ${ }^{1}$ Fatemeh Salehi², Laszlo Nadai ${ }^{1}$, Szell Karoly ${ }^{3}$, Nima E. Gorji ${ }^{4, *}$ \\ ${ }^{1}$ Kalman Kando Faculty of Electrical Engineering, Obuda University, Budapest, Hungary; \\ amir.mosavi@kvk.uni-obuda.hu,nadai@uni-obuda.hu \\ 2 School of Physics, Iran University of Science and Technology, Tehran, Iran; \\ Fatemehsalehi1984@gmail.com \\ ${ }^{3}$ Alba Regia Technical Faculty, Obuda University, Szekesfehervar, Hungary; \\ szell.karoly@amk.uni-obuda.hu \\ ${ }^{4}$ Optoelectronics Research Group, Faculty of Electrical and Electronics Engineering, Ton Duc \\ Thang University, Ho Chi Minh City, Viet Nam \\ ${ }^{*}$ Correspondence: nimaegorji@tdtu.edu.vn
}

\begin{abstract}
A novel mathematical model is developed to calculate the temperature distribution on the surface and bulk of a steel plate under the laser hardening process. The model starts with the basic heat equation then it is developed into a volumetric form and is connected to the various solid existing phases. The proposed model is based on three influencing parameters of the laser hardening process which are the velocity of the laser spot and irradiation time. The results are compared with the available experimental data reported in the literature. The volumetric model provides an assessment of temperature distribution in both the vertical and horizontal axis. Laser irradiation at sufficiently high fluence can be used to create a solid-state phase change on the surface. Primary calculations show that the temperature profile has a Gaussian distribution in horizontal $\mathrm{x}$ and $\mathrm{y}$-axis and presents an exponentially decreasing in the horizontal and vertical depth directions.
\end{abstract}

Keywords: Laser hardening, Temporal-temperature profile, Solid phase transformation, heat treatment.

\section{INTRODUCTION}

Laser hardening is becoming a unifying process in various industrial production lines and sectors ranging from automotive processing lines to die/mold industrial sectors. Two key parameters to ensure the process is completed by success are: controlling the surface temperature of the medium and the thickness of the hardened layer [1]. The temperature of the surface requires a continuous verification during the applying the process and even after completing the process which prevents surface from melting but maintains the temperature uniform distribution [2]. AlMangour group have developed a simulation model to predict the evolution of temperature on surface and thermal behaviors of the molten pool beneath various volumetric laser energy densities during the selective laser melting [3]. Sarkar et al. have developed the simulation analysis of temperature and surface hardening of low carbon thin steel sheets using $\mathrm{Yb}$-fiber laser [4]. These analysis show that temperature increase over time and into depth for different laser processing conditions is quite effective on depth hardness of the plate. Bojinovic et al. have developed a simulation study on temperature distribution of a steel plate and it's changing rate on austenite kinetics in laser hardening process [5]. They reported that the effect of different heat inputs a similar peak in temperature distribution is obtained on the sample surface. However, a deeper area of increased hardness is formed only when smaller laser beam power and velocity are applied. A similar conclusion was also given by a hardness study by Saeidi et al. [6]. They suggested that using high-energy focused laser beam and very short times at elevated temperature gives a higher quality of steel laser sintering. Jiang et al. have shown that pulse energy is the main significant factor affecting the diameter and depth of transformation hardening zone in steel laser hardening [7].

$$
Q(x, z, t)=Q_{0} \exp \left(\frac{-(x-V t)^{2}}{N_{b}^{2} N_{s}^{2}-\left(Z-Z_{m a x}\right)^{2}}\right)
$$

Where $\mathrm{N}_{b}$ and $\mathrm{N}_{\mathrm{s}}$ are surface and bulk source concentration factors for the medium under study at the area under the laser beam. The surface and bulk concentration factors are also defined by stress factor (cm). The current presentation aims to develop a thermos-physical approach for modeling the temperature distribution at different stage of laser hardening known as hardening process by e-beam applied on carbon steels which is applied on the plate surface by a e-beam with relativistic beam velocities. We have already developed a uniform/non-uniform model for temperature distribution in solar cells with semiconducting layers [8]. Here we model the temperature profile a Steel plate $(\mathrm{H} 13)$ with $3 \mathrm{D}$ shape under laser hardening process as schematically shown in Fig. 1. We must assume that the laser produces relativistic flow of electrons by heating the surface by (uniformly) moving the laser in X-axis on the surface. Clearly, heat will also uniformly melt the surface along Y-axis. Thus, we can reduce the problem to a $2 \mathrm{D}$ temperature profile (XZ-plane) on the surface. Considering the temperature profile in Z-axis is reasonable sine we assumed the relativistic electrons are produced under laser hardening and they will have a higher penetration depth than the non-relativistic electrons i.e. with room temperature velocity. Therefore, we can use the heat equation in volumetric form where $\mathrm{Q}_{0}$ is the maximum flow rate density $\left(\mathrm{W} / \mathrm{cm}^{2}\right) ; \mathrm{N}_{\mathrm{s}}$ is the surface source concentration factor $(\mathrm{cm}) ; \mathrm{N}_{\mathrm{b}}$ is the bulk source concentration factor $(\mathrm{cm})$ and $Z_{\max }$ is the depth at 
which the energy released is a maximum $(\mathrm{cm})$. We selected $\mathrm{Q}_{0}=2.3 \times 10^{5} \mathrm{~J} / \mathrm{cm}^{2} . \mathrm{s}$ and $\left.\mathrm{V}=1 \mathrm{~cm} / \mathrm{s}\right)$. Normally, the maximum energy is related to the penetration depth (l) of the generated electrons via $Z_{\max }=4$. The general heat conduction equation is time and position dependent as following:

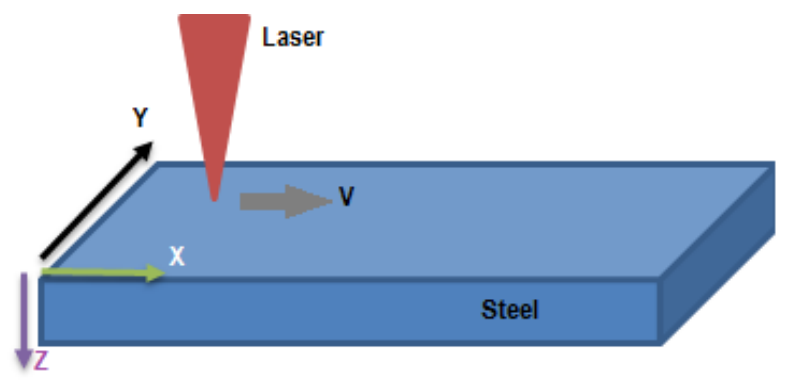

Fig. 1 - Schematic of a laser hardening process and a steel with parallelepiped shape.

$$
-k \nabla^{2} T(t, r)+Q(r)=c_{p} \rho \frac{d T(t, r)}{d t}
$$

where $\mathrm{k}$ is the thermal conductivity of the material as a function of temperature $[\mathrm{W} /(\mathrm{m} . \mathrm{K})]$, cp is the specific heat $[\mathrm{J} /(\mathrm{kg} . \mathrm{K})]$ and $\mathrm{Q}$ is the heat generation rate. Then, we may extend the Eq. (1) in Cartesian coordinate system $\mathrm{x}, \mathrm{y}, \mathrm{z}$ in the steady state condition $(\mathrm{dT}=0)$,

$$
\mathrm{c}(\mathrm{T}) \rho(T)\left(\frac{\partial T}{\partial t}\right)=\frac{\partial}{\partial x}\left(\frac{k \partial T}{\partial x}\right)+\frac{\partial}{\partial z}\left(\frac{k \partial T}{\partial z}\right)+Q
$$

where $\mathrm{Q} *$ is given by Eq. (1), $\mathrm{k}=1.22 \mathrm{~W} /(\mathrm{cm} . \mathrm{s.K})$ is the thermal conductivity as a function of temperature [W/(m.K)], c(T) is the specific heat [J/(kg.K)]. The heat capacity and density are temperature dependent and can be approximated by polynomials:

$$
\begin{aligned}
& C(T)=a_{1}+b_{1} T+c_{1} T^{2}+d_{1} T^{3} \\
& \rho(T)=a_{2}+b_{2} T
\end{aligned}
$$

the ai, bi, ci, and di can be obtained using the value of heat capacity and density of materials before and after melting temperature in solid and liquid phases. Clearly in laser hardening process we will have domains of double phases with both liquid and solid phases especially when the medium under study is a single material (e.g. Steel only). Therefore, the following relation must be valid at the molten pool where all the liquid, solid and particles are present,

$$
\emptyset_{\text {liq }}+\emptyset_{\text {sol }}+\emptyset_{\text {par }}=1
$$

This equation can be discussed in different conditions:

$$
\begin{gathered}
\emptyset_{\text {liq }}=1-\emptyset_{p} \text { for } T \geq T_{\text {liq }} \\
\emptyset=0 \text { for } T \leq T_{\text {liq }} \\
\text { genearlly valid } \emptyset_{\text {par }}<<\emptyset_{\text {liq }}+\emptyset_{\text {sol }}
\end{gathered}
$$

Let define the temperature of every solid and liquid phase by linear approximations taking into account the presence of particles in the pool,

$$
\begin{aligned}
T_{\text {sol }} & =T_{m}-\partial T * \phi_{\text {par }}-\Delta T_{m} \\
T_{\text {liq }} & =T_{m}-\partial T * \phi_{\text {par }}-\Delta T_{m}
\end{aligned}
$$

where $\partial \mathrm{T}$ and $\Delta \mathrm{T}_{\mathrm{m}}$ are unknown parameters to be defined by the state diagrams and represent small temperature interval from the melting point (temperature) in a double phase system. The two equations presented in Eq. (6) are connected by volume fraction of liquids exist in the molten pool according to "Phase Zone" theory presented in Ref. [9],

$$
\phi_{l i q}=1-\left(\frac{T_{l i q}-T}{T_{l i q}-T_{s o l}}\right)^{2}
$$

The above connection point is valid since we are dealing with a two phase system and thus we must take into account the volume fraction of both solid and liquid phases in the pool. In addition, the particles are produced under irradiation and do exist in the process. The boundary conditions of the above equations are as following:

$$
\begin{aligned}
& \text { at } t=0: T=T 0 \\
& \text { at } x=0, \infty: \frac{\partial T}{\partial x}=0 \\
& \text { at } z=0: k \frac{\partial T}{\partial x}=\epsilon_{0} \sigma\left(T^{4}-T_{0}^{4}\right) \\
& \text { at } z=\infty: \frac{\partial T}{\partial z}=0, \emptyset_{\text {par }}=0 .
\end{aligned}
$$

where T0 is the initial temperature and the term $\varepsilon_{0} \sigma\left(\mathrm{T}_{4}-\mathrm{T}_{04}\right)$ is heat transfer described by Stefan-Boltzmann equation. We remember that also the heat capacity is variable at near-melting temperatures (for Steel 20) thus we can take the effective heat capacity by using two-phase zone theory [10]:

$$
(C \rho)_{e f f}=(C \rho)+L_{m} \rho_{b, s} \frac{\partial \emptyset}{\partial T}
$$

where $L_{m}$ is the melting heat capacity. We can write the heat capacity based on the volume fraction of solid and liquid phases [4]:

$$
(\mathrm{C} \rho)=(\mathrm{C} \rho)_{\text {sol }}\left(1-\emptyset_{\text {liq }}\right)+(\mathrm{C} \rho)_{l i q} * \emptyset_{\text {liq }}
$$

\begin{tabular}{|l|c|c|c|c|}
\hline \multicolumn{1}{|c|}{$\#$} & $\mathrm{ai}$ & $\mathrm{b}_{\mathrm{i}}$ & $\mathrm{Ci}$ & $\mathrm{d}_{\mathrm{i}}$ \\
\hline $\mathrm{C}_{\text {sol }}$ & 0.130 & $\begin{array}{c}0.01 \times \\
10^{-3}\end{array}$ & $4.04 \times 10^{-9}$ & - \\
\hline $\begin{array}{l}\mathrm{C}_{\text {sol }} \text { for } \\
280<\mathrm{T}<1040 \mathrm{~K}\end{array}$ & 0.42 & 0.005 & $-8.65 \times 10^{-6}$ & $5.16 \times 10^{-9}$ \\
\hline $\begin{array}{l}\mathrm{C}_{\text {sol }} \text { for } \\
1040<\mathrm{T}<1800 \mathrm{~K}\end{array}$ & 115.6 & -0.312 & $3.15 \times 10^{-4}$ & $-1.41 \times 10^{-7}$ \\
\hline$\rho_{\text {sol }}$ & 19.3 & $33 \times 10^{-6}$ & - & - \\
\hline \multicolumn{5}{|c|}{$(\mathrm{C} \rho)_{\text {sol }}=(\mathrm{C} \rho)_{\text {sol }}\left(1-\emptyset_{\text {par }}\right)+(\mathrm{C} \rho)_{\text {par,liq }} * \emptyset_{\text {par }}}$. \\
\hline
\end{tabular}

Table 1: The value of the coefficients given in Eq. (3) \& (4) extracted from Ref. [4].

\section{Modeling Results and Discussion}

The above presented set of equations are numerically 
solved using the value of the parameters given in Table I and the boundary conditions given in Eq. (9). We calculated the temperature profile of the Steel plate at different times of the laser hardening process as well as the temperature distribution at different area of the processed point such as heated zone, heat-affected zone, and melt pool. Table I presents the materials properties of Steel as presented in literature. We note that our modeling is based on assuming the laser produces electron flow with high velocity or taking the laser photos as particles with high velocity which can change the phase of the materials within a very short time interval. In any case, in a short time after applying the laser on the Steel plate the melting process becomes semi-stationary as we also assumed to be the case for solving the Eq. (2). This assumption allowed us to set Tm max temperature in a molten pool and the surrounding heated area. As given in Eq. (1) this maximum temperature (related to the generated heat) depends on the velocity of the laser moving on the surface of the plate. Also it depends on the processed material's properties as set in $\mathrm{N}_{\mathrm{b}}$ and $\mathrm{N}_{\mathrm{s}}$ parameters. Fig. 1 shows the distribution of temperature along the $\mathrm{x}$-axis of the Steel plate.

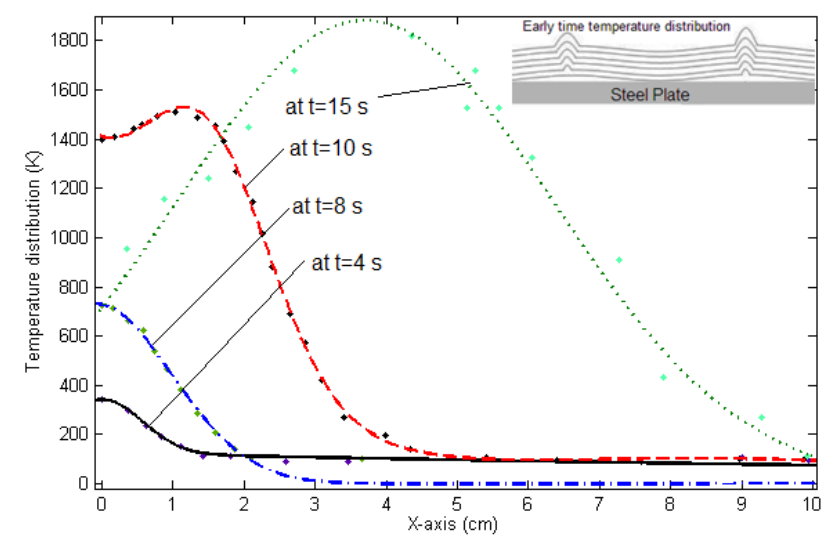

Fig. 2 - Temperature distribution at different times along the $\mathrm{x}$-axis by starting laser tip to move over the specimen surface from the very left to very right direction. The data points are taken from [4]. A good fit is obtained at different times of the laser irradiation with measured data. The exponential trend of the temperature distribution tends to become Gaussian at later times. Inset is schematic of temperature distribution on surface at $\mathrm{t}=4 \mathrm{~s}$.

The laser tip is starting (on the plate surface) from the very left and probing continues to right with speed of $\mathrm{V}=0.5 \mathrm{~cm} / \mathrm{s}$. We have calculated the temperature distribution at different times of laser shining e.g. $\mathrm{t}=4 \mathrm{~s}, 8$ $\mathrm{s}, 10 \mathrm{~s}$ and $15 \mathrm{~s}$. It is observed that the temperature distribution is like an exponential distribution within the initial time interval of irradiation from $t=0-4 \mathrm{~s}$ and it becomes more intense peak a bit later at $\mathrm{t}=8 \mathrm{~s}$. the slope of the temperature variation along the $\mathrm{x}$-axis becomes more steep at $t=8 \mathrm{~s}$. This trend continues to be sharper at $\mathrm{t}=10 \mathrm{~s}$ and becomes slightly mild at $\mathrm{t}=15 \mathrm{~s}$. Temperature peak shifts to right at $\mathrm{t}=10 \mathrm{~s}$ and that Gaussian feature becomes rather significant at $\mathrm{t}=15 \mathrm{~s}$. Still we see a bigger shift to right for $\mathrm{t}=15 \mathrm{~s}$ which means that the temperature tends to get a symmetric distribution by moving the laser to right even if the laser is getting away from the center at the very left.
Figure 2: Temperature distribution at different times along the $\mathrm{x}$-axis by starting laser tip to move over the specimen surface from the very left to very right direction. The data points are taken from [4]. A good fit is obtained at different times of the laser irradiation with measured data. The exponential trend of the temperature distribution tends to become Gaussian at later times. Inset is schematic of temperature distribution on surface at $\mathrm{t}=4 \mathrm{~s}$.

We also calculated the distribution of temperature via $\mathrm{z}$-axis from the top surface down to interior bulk or depth of the plate. Fig. 3 shows the temperature changes across the z-axis. The peak occurs at the very surface and reduces to lower temperatures by going down to the interior bulk. However, temperature tends to keep the Gaussian shape even until $\mathrm{t}=8 \mathrm{~s}$. This symmetric trend disappears slowly by time and at $\mathrm{t}=10 \mathrm{~s}$ it takes a mild exponential feature and it is competed at $\mathrm{t}=15 \mathrm{~s}$ to become fit with a double term exponential function. We note that all the peaks are very close to the surface which means a less impact of temperature to the interior bulk. That recalls a heating pool is more probable to occur at very surface of slightly underlying surface levels. The peak is shifting to left in this case which is again in contrast to right-shift of $x$-axis calculations. The energy release at depth of the plate is forming the melting pool and also recommends a different energy loss rates from the surface level. We believe this energy loss could most probably be because of heat conduction from the interior bulk of the plate and partially because of emission of rays releasing the energy from the top side (surface). Sarkar et al. [4] have also experimentally and by simulation proved that for longer laser interaction time the plate surface is cannot cool down unless from the surface because heat release along depth too is not effective in plate with small thickness. The implication of this results is that if one wishes to increase the temperature penetration into depth, the hardening process must go with slower laser speed. The inset of Fig. 3 is a schematic of how the temperature distribution occurs on the plate surface at early stage of laser hardening process. The peak is relevant to first time interval of laser irradiation. Laser hardening process was also well documented in Ref. [12].

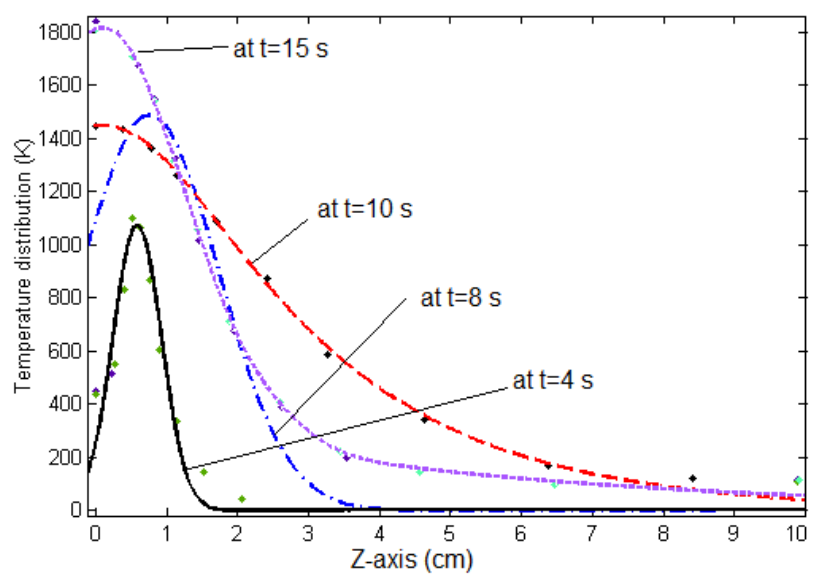

Fig. 3 - Temperature distribution at different times in zaxis (towards depth from surface) by starting laser tip to move over the specimen surface from the very left to very right direction. The data points are taken from [4]. A good fit is obtained 
a different times of the laser irradiation with measured data. In contrast to $\mathrm{x}$-axis distribution shown in Fig. 2 the temperature distribution is Gaussian at the initial times and tends to exponential trend at later times.

Figure 3: Temperature distribution at different times in z-axis (towards depth from surface) by starting laser tip to move over the specimen surface from the very left to very right direction. The data points are taken from [4]. A good fit is obtained at different times of the laser irradiation with measured data. In contrast to $\mathrm{x}$-axis distribution shown in Fig. 2 the temperature distribution is Gaussian at the initial times and tends to exponential trend at later times.

\section{Conclusion}

A theoretical approach was developed to model the temperature distribution on the surface and depth of a Steel plate which is under surface treatment with laser flow. In order to facilitate our modeling, we proposed that the laser photons flow can be assumed like a flow of electrons irradiated on the surface with a determined flux. This assumption helps us to identify how many particles (per surface or volume) are in interaction with the laser. The crystallization process is clearly affected by temperature distribution at different times of laser processing via $\mathrm{x}$-axis and $\mathrm{z}$-axis and the melting pool created around the surface can represent the temperature distribution and its impact on this crystallization process. The modeling is fitted with experimental data reported in the literature. The temperature distribution along the $\mathrm{x}$-axis and $\mathrm{z}$-axis are contrary developed by time where the peak shift and slope of temperature distribution as well symmetry of the distribution is in contrast to each-other. While the temperature distribution tends to peak at the middle of the plate, it is tending to peak at the surface and not in the bulk part. The model can be extended to find the interfere of the impurities and other alloys present in the medium as well. Also, we can simulate the temperature distribution by modeling the phase and crystallization out of an extended model presented here i.e. as a three-dimensional temperature profiling $[13,14,15]$.

\section{References}

1. A. G. Knyazeva, O. N. Kryukova, Fiz. Mezomekh., 7:2 (2004) 81-89.
2. H. G. Woo, H. S. Cho, "Three-dimensional temperature distri ᄀbution in laser surface hardening processes", Proc. Instn. Mech. Engrs., Vol 213 (1999) Part B.

3. B. AlMangour, D. Grzesiak, J. Cheng, Y. Ertas, "Thermal behavior of the molten pool, microstructural evolution, and tribological performance during selective laser melting of TiC/316L stainless steel nanocomposites: Experimental and simulation methods, Journal of Materials Processing Technology, 257 (2018) 288-301.

4. S. Sarkar, M. Gopinath, Sh. S. Chakraborty, B. Syed, A. K. Nath, Analysis of temperature and surface hardening of low carbon thin steel sheets using Yb-fiber laser", Surface \& Coat $\neg$ ings Technology, 302 (2016) 344-358.

5. M. Bojinović, N. Mole, B. Štok, 'A computer simulation study of the effects of temperature change rate on austenite kinetics in laser hardening", Surface \& Coatings Technology, 273 (2015) 60-76

6. K. Saeidi, X. Gao, Zhong, Z.J. Shen, "Hardened austenite steel with columnar sub-grain structure formed by laser melting”, Materials Science \& Engineering A, 625 (2015) 221-229.

7. J. Jiang, L. Xue, Sh. Wang, 'Discrete laser spot transformation hardening of AISI O1 tool steel using pulsed Nd:YAG laser", Surface \& Coatings Technology, 205 (2011) 51565164 .

8. M. D. Perez, N. E. Gorji, "Modeling of temperature profile, thermal runaway and hot spot in thin film solar cells," Mater. Sci. Semicond. Process., 41 (2016) 529-534.

9. V. T. Borisov theory of two phase zone in metal ingots", Metallurgia, Moscow (1987).

10. A. Bergen, B. Adl-Zarrabi, C. E. Hagentoft, 'Determination of specific heat capacity by transient plane source", Frontiers of Architectural Research, 2 (2013) 476-482.

11. J. Venema, J. Hazrati, D.T.A. Matthews, R.A. Stegeman, A.H. Boogaar, "The effects of temperature on friction and wear mechanisms during direct press hardening of $\mathrm{Al}-\mathrm{Si}$ coated ultra-high strength Steel, Wear, Volumes 406-407, 15 July 2018, Pages 149-155.

12. A.K.NathS. Sarkar, 'Chapter 11 - Laser Transformation Hardening of Steel, Advances in Laser Materials Processing (Second Edition)", Technology, Research and Applications, Woodhead Publishing Series in Welding and Other Joining Technologies, 2018, Pages 257-298.

13. J. Weber, 'Independent metering systems", International Journal of Hydromechatronics, Volume 3, Issue 1, p. 107-125 (2018).

14. Zh. Wang, Zh. Xie, W. Huang, 'A pin-moment model of flexoelectric actuators", International Journal of Hydromechatronics, Volume 3, Issue 1, p. 72-90 (2018).

15. J.-P. Henderson, A. Plummer, N. Johnston, 'An electrohydrostatic actuator for hybrid active-passive vibration isolation", International Journal of Hydromechatronics, 3, Issue 1, p. 47-71 (2018). 